\title{
Methods and models of ensuring reliability of district heating systems with prosumers
}

\author{
Ivan Postnikov ${ }^{1, *}$, Valery Stennikov ${ }^{1}$ \\ ${ }^{1}$ Melentiev Energy Systems Institute, 130 Lermontov str., Irkutsk, Russia
}

\begin{abstract}
The paper is concerned with the statement of a district heating systems reliability problem. The reliability is planned to be ensured through the optimal distribution of reliability parameters among components of system, considering the capabilities of the prosumer to supply part of load by their heat sources. The methods and models are suggested to solve this problem. They are based on the theory of random processes, theory of hydraulic circuits and basic laws of cogeneration. The case study results obtained using the developed technique are presented. A potential economic benefit of involving the prosumer is demonstrated in the case study. The conclusions and directions for further research are formulated.
\end{abstract}

\section{Introduction}

One of the major principles of a contemporary energy systems paradigm is an increase in the role of consumer in energy supply. This principle is implemented in the framework of a prosumer (producer-consumer, consumer that have self-generation) concept which implies control and optimization of the consumer load curve by using the consumer-owned sources and storage devices with a view to enhance the efficiency and reliability of both the consumer and the entire energy system.

The authors of [1-7] present the studies on various aspects of operation and control of prosumer within the electric power systems. Although, the issues of prosumer operation in district heating systems (DHS) are raised much more rarely $[8,9]$, they are relevant and important since DHS are the largest fuel consumers in many countries.

Nowadays, modern DHS are experiencing an increase in heat loads, which causes some problems related to the fulfilment of volume and reliability requirements in heat supply. The creation of the prosumers with their heat sources (HS) will provide additional capacity and time reserve which will reduce the load of district HS and thus improve the quality and reliability of both heat supply to consumers and operation of the system as a whole.

Thus, one of the key prosumer goals within the DHS is to increase reliability of the system operation. The methodological problems to be solved to achieve this goal are mainly traditional for the analysis and synthesis of reliability of energy system as a whole and DHS, in particular. At the same time, the involvement of prosumer in the DHS operation introduces new aspects in these problems and requires new methodological approaches to solve them.

\section{Methods and models to ensure reliability of heating systems with prosumers}

\subsection{Statement of the problem}

The development of methods for reliability analysis and optimization in the DHS with prosumer generates two main lines of investigations. The first line is related to an analysis and optimization of heat supply reliability, considering the prosumer functions in the system, provided the quantity, nodes and capacity of the prosumer sources are specified. The second line represents the problem aimed at determining the amount of prosumers, their connection points and capacities.

This paper is focused on the first reliability problem which implies the determination of the values of reliability parameters of the DHS components (failure and restoration rates) that ensure the required reliability level of heat supply to consumers, including prosumers (considering their additional reserve), at minimum cost of reaching these indices within their feasible range.

Two main reliability indices are assumed to assess the level of heat supply reliability [10]. These are failure free operation probability $R_{j}$ (FOP) and availability factor $K_{j}(\mathrm{AF})$. These indices are determined for each consumer $j \in J$, where $J$ is a set of consumers in the system (including prosumers). The standard values of these indices are denoted by $R_{\mathrm{o} j}$ and $K_{\mathrm{o} j}$.

The stated problem is solved with respect to these reliability indices according to a technique consisting of 4 stages: 1) determine relationships between the average reliability parameters of DHS components; 2) model post-emergency conditions of the DHS; 3) model the

Corresponding author: postnikov@isem.irk.ru 
functions of the prosumers; 4) formalize the problem of determine optimal reliability parameters of the DHS components.

\subsection{The relationship between the average reliability parameters of DHS components}

Average reliability parameter of components is taken to mean their failure or restoration rate that preliminarily has the same value for these components, which provides the required level of reliability indices. These parameters are determined by the formulas intended for the calculation of nodal reliability indices of DHS [10], Rossander equation that determines annual heat load curves of consumers [11], and some basic laws of district heating and thermal physical processes involved in DHS [12].

Based on the theoretical studies we determined the following relationship between the average reliability parameters of DHS components (failure rate and restoration rate), considering meeting the conditions of FOP and AF:

$$
\begin{aligned}
\bar{\lambda}_{j}=\frac{1}{\tau_{\mathrm{o}}}\left(\ln \frac{1}{R_{\mathrm{o} j}}\right) & \times\left(1-N_{s}\left(1-K_{\mathrm{o} j}\right) / \sum_{s \in E} L_{s}^{1 / \sigma_{j}}\right)^{-1} \times \\
& \times\left(\sum_{s \in E} M_{s}^{1 / \sigma_{j}}\right)^{-1} ;
\end{aligned}
$$

where

$$
\begin{gathered}
L_{s}=\frac{1}{1-\omega_{j}} \times \\
\times\left[1-\frac{1}{q_{\mathrm{o} j}}\left(q_{s j}+\varphi_{j}\left(t_{s j}-\frac{C_{1}-C_{2} \exp B_{j}}{C_{3}\left(1-\exp B_{j}\right)}\right)\right)\right] \\
M_{s}=\frac{1}{1-\omega_{j}}\left(1-\bar{q}_{s j}+\frac{\varphi_{j} t_{s j}}{q_{\mathrm{o} j}}-\varphi_{j} \frac{C_{1}-C_{2} \exp B_{j}}{C_{3} q_{\mathrm{o} j}\left(1-\exp B_{j}\right)}\right) ; \\
C_{1}=t_{\mathrm{o} j}\left(1-\bar{q}_{s j}\right), C_{2}=t_{j \min }-t_{\mathrm{o} j} \bar{q}_{s j}, C_{3}=1-\bar{q}_{s j} ; \\
\bar{q}_{s j}=q_{\mathrm{o} j} / q_{s j}, s \in E, j \in J \\
B_{j}=1 /\left(\varepsilon_{j} \bar{\mu}_{j}\right), j \in J .
\end{gathered}
$$

Here: $\bar{\lambda}_{j}$ and $\bar{\mu}_{j}$ - average failure and restoration rates for consumer $j$, respectively, $1 / \mathrm{h} ; \tau_{\mathrm{o}}$ - time instant corresponding to a total number of hours of the considered (heating) period, $\mathrm{h} ; N_{s}$ - the quantity of system states; $s$ - number of system state; $E$ - a set of system states; $\omega_{j}, \sigma_{j}$ - irregularity factors of heat load curve of consumer $j[11] ; q_{\mathrm{o} j}-\operatorname{design}$ (maximum) heat load of consumer $j, \mathrm{GJ} / \mathrm{h} ; q_{s j}-$ level of heat supply to consumer $j$ in system state $s, \mathrm{GJ} / \mathrm{h} ; \varphi_{j}-$ coefficient of specific heat losses for consumer $j$, $\mathrm{GJ} /\left(\mathrm{h}^{\circ} \mathrm{C}\right) ; t_{s j}$ - current (actual) internal air temperature for consumer $j$ in system state $s,{ }^{\circ} \mathrm{C} ; \bar{q}_{s j}-$ relative heat supply to consumer $j$ in system state $s, \mathrm{GJ} / \mathrm{h} ; t_{\mathrm{o} j}$ - design temperature of internal air for consumer $j,{ }^{\circ} \mathrm{C}$; $t_{j \text { min }}$ - minimum admissible temperature of internal air for consumer $j,{ }^{\circ} \mathrm{C} ; \varepsilon_{j}$ - coefficient of thermal energy storage for consumer $j, \mathrm{~h} ; L_{s}, M_{s}, C_{1}, C_{2}, C_{3}, B_{j}-$ assumed abbreviations of expressions.

\subsection{Modeling of prosumer functions}

The prosumer functions in the emergency conditions leading to limitation or total termination of heat supply are aimed at eliminating heat undersupply by their HS, and increasing time reserve. These properties of the prosumer are considered in the calculation of indices $\bar{q}_{s j}$ and $B_{j}$. To this end, the following expressions are added to equations (5) and (6):

$$
\begin{gathered}
\bar{q}_{s j}=q_{\mathrm{o} j} /\left(q_{s j}^{\mathrm{sys}}+q_{s j}^{\prime}\right), s \in E, j \in J \\
B_{j}=1 /\left[\left(\varepsilon_{j}+\Delta \varepsilon_{j}\right) \bar{\mu}_{j}\right], j \in J
\end{gathered}
$$

where $q_{s j}^{\text {sys }}$ - part of heat load of consumer $j$, supplied from the system (by district HS) in system state $s, \mathrm{GJ} / \mathrm{h}$; $q_{s j}^{\prime}$ - capacity of HS for consumer $j$ (for prosumer) in system state $s, \mathrm{GJ} / \mathrm{h} ; \Delta \varepsilon_{j}$ - additional passive time reserve of prosumer (caused by the use of their heat generators and/or heat storage devices), $h$.

Index $q_{s j}^{\prime}$ can be fixed and correspond to a rated (required) value of capacity of the prosumer HS. This index can be variable and take into account the components failures decreasing the performance of the HS. Here, the corresponding failures should be added to a set of the system states. In this case, decision should be made based on an analysis of initial characteristics of equipment reliability: if the values of failure flow for the HS equipment of the prosumer are much lower than for the DHS equipment, then the failures at prosumer HS can be neglected, and $q_{s j}^{\prime}$ can be assumed to be fixed according to their required performance under specified emergency heat supply to consumers.

\subsection{Modeling of post-emergency conditions in heating systems}

Post-emergency hydraulic conditions are determined by steady flow distribution in the heat network (HN) after disconnection of an emergency component. The hydraulic conditions are calculated by the methods of theory of hydraulic circuits [13]. The nodal form of writing the model of flow distribution (hydraulic conditions) in the network is represented by a matrix and has the form [13]:

$$
\mathbf{A x}_{s}=\mathbf{q}_{s}, s \in S
$$




$$
\begin{gathered}
\overline{\mathbf{A}}_{s}^{\mathrm{T}} \mathbf{p}_{s}=\mathbf{h}_{s}-\mathbf{H}_{s}, s \in S \\
\mathbf{S X}_{s} \mathbf{x}_{s}=\mathbf{h}_{s}, s \in S
\end{gathered}
$$

where: $\mathbf{A}_{s}$ - incidence matrix of linearly independent nodes in the network under emergency system state $s$ (considering failure of some component); $\overline{\mathbf{A}}_{s}^{\mathrm{T}}-$ full transposed node-branch incidence matrix; $\mathbf{x}_{S}-$ vector of heat carrier flow rates in the network sections (branches) under emergency system state $s, \mathrm{t} / \mathrm{h} ; \mathbf{q}_{s}-$ vector of flow rates at network nodes under emergency system state $s, \mathrm{t} / \mathrm{h} ; \mathbf{p}_{s}$ - vector of nodal pressures of $\mathrm{HN}$ under emergency state $s, \mathrm{~mm} \mathrm{wc} ; \mathbf{h}_{s}-$ vector of heat losses in the sections under the emergency system state $s, \mathrm{~mm}$ wc; $\mathbf{H}_{s}$ - vector of operating heads at sources in the emergency system state $s, \mathrm{~mm} w c ; \mathbf{S}, \mathbf{X}_{s}$ - diagonal matrices of coefficients of hydraulic resistance of sections, $\mathrm{m} /\left(\mathrm{h}^{2} \mathrm{t}^{2}\right)$, made up from the values of hydraulic resistances of sections and absolute values of flow rates in them, $\mathrm{t} / \mathrm{h}$.

Modeling of an emergency situation in some state $s$ of the system is performed by excluding a component whose failure corresponds to this state from the calculation DHS scheme.

\subsection{Formalization of a problem of determination of optimal reliability parameters of DHS components}

The values of average reliability parameters determined by expressions (1)-(6) are distributed among the system components according to the following equations of conservation of the sum of system state probabilities:

$$
\begin{aligned}
& \bar{\lambda}_{j} \sum_{s \in E} p_{s}=\sum_{n \in N} \sum_{s \in E(n)} \lambda_{n} p_{s}, j \in J ; \\
& \bar{\mu}_{j} \sum_{s \in E} p_{s}=\sum_{n \in N} \sum_{s \in E(n)} \mu_{n} p_{s}, j \in J ;
\end{aligned}
$$

where: $p_{s}$ - probability of system state $s ; n$-number of system component; $N$ - a set of system components; $E(n)$ - is a subset of system states for which the system can transition because of failure or restoration of component $n ; \lambda_{n}, \mu_{n}$ - failure or restoration rates of component $n, 1 / \mathrm{h}$.

The probabilities of system states are determined by solving the equations of Markov process that describes a sequence and structure of events that characterize the DHS operation. The Markov models are a justified and universal tool for the assessment of reliability of restorable systems [14-17].

A stationary Markov model of DHS operation can be represented by the following system of linear equations:

$$
\begin{gathered}
p_{s}\left(\sum_{n \in N(s)} \lambda_{n}+\sum_{n \in N(s)} \mu_{n}\right)= \\
=\sum_{z \in E(s)}\left(\sum_{n \in N(z)} p_{z} \lambda_{n}+\sum_{n \in N(z)} p_{z} \mu_{n}\right), s \in E ;
\end{gathered}
$$

where: $p_{z}$ - probability of the system state $z$ (division of state into $s$ and $z$ is necessary to write the system of equations of random process); $N(s)$ - subset of system components whose failure or restoration corresponds to a direct transition of the system from state $s$ to some other state $z ; N(z)$ - subset of system components whose failure or repair corresponds to a direct transition of the systems from state $z$ to some other state $s ; E(s)-$ subset of the system states from which the system can transition to state $s$.

The rationale of using the stationary Markov model as well as the other aspects of using the tool of Markov random processes in the problems of DHS reliability are considered in more detail in [14, 18-22].

According to the problem stated in 2.1, the objective function of DHS reliability optimization is expressed as a sum of costs of ensuring the values of reliability parameters of the DHS components:

$$
F_{\mathrm{obj}}=\sum_{n \in N} f_{n \lambda}\left(\lambda_{n}\right)+\sum_{n \in N} f_{n \mu}\left(\mu_{n}\right)
$$

where $f_{n \lambda}\left(\lambda_{n}\right), f_{n \mu}\left(\mu_{n}\right)$ - cost functions of ensuring reliability parameters of components, i.e. their failure and restoration rates, respectively, rub. The form of functions (15) and their quantitative parameters are determined by the methods of approximation on the basis of an analysis of the actual data on the cost of equipment with different reliability characteristics, costs of installing the backup components; costs of establishing and maintaining the emergency and restoration services (ERS) and other measures capable to improve the reliability.

Technically possible values of reliability parameters of the components are specified by the following constraints:

$$
\begin{aligned}
& \lambda_{n}^{\min } \leq \lambda_{n} \leq \lambda_{n}^{\max }, n \in N ; \\
& \mu_{n}^{\min } \leq \mu_{n} \leq \mu_{n}^{\max }, n \in N .
\end{aligned}
$$

Thus, the optimization problem of component reliability of DHS considering prosumers lies in the following:

- conditions (1)-(6), that determine the relations between average reliability parameters of DHS components considering the prosumer functions;

- conditions (9)-(11) according to which the levels of heat supply under different system states are calculated; - conditions (12) and (13) that determine the principles of allocating the average reliability parameters among the system components; 
- constraints (16) and (17) on the reliability parameters of components;

Nonlinearity of considered optimization problem is connected firstly with function (15), which, as a rule, is a power function. Solution of such problems is based on method of coordinate-wise relaxation (or method of coordinate-wise descent) with use of simple iteration method within solve cycle. The GAMS software package is used as a solver.

\section{Case study and analysis of results}

Consideration is given to DHS scheme presented in Fig. 1-1. The scheme consists of two district heat sources (HS1 and HS2), seven consumers (nodes 1-7) and a looped HN consisting of 18 sections (components). One of the consumers is a prosumer (P6) with a load of 400 $\mathrm{GJ} / \mathrm{h}$, and a HS with a capacity of $200 \mathrm{GJ} / \mathrm{h}$. An aggregate scheme of components for both district HS is demonstrated in Fig. 1-2 and consists of: a boiler 19, a turbine 20, network heaters 21 and $23 \mathrm{GJ} / \mathrm{h}$ and network pumps 22 and 24. The indicated numbers of components correspond to HS1, the numbers of components for HS2 are assigned in the order from 25 to 30 . Technical characteristics of the HN components, consumer loads and capacities of HS are given in Table 1 .

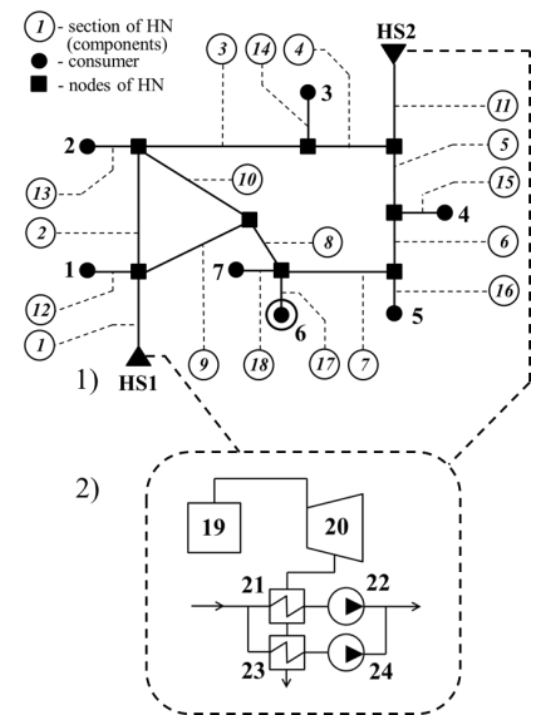

Fig. 1. A scheme of DHS: 1) a general scheme of the DHS; 2) a simplified scheme of HS

The calculation scheme of a considered DHS is constructed according to a comprehensive approach to the research into the reliability of heat supply [18-20] as a joint structure integrating the components of the schemes of $\mathrm{HS}$ and $\mathrm{HN}$ and is given in Fig. 2. The components 1-18 are network sections, components 1930 correspond to HS1 and HS2.

The random process of DHS operation is modeled for the following conditions: each component can be in two states - operable and failed, and the flow of events within one subsystem (HN, HS1 and HS2) is the simplest. The latter condition suggests a simultaneous failure of components only from different subsystems of
DHS. Here we will confine ourselves to the consideration of the state when no more than 2 components fail at once. Thus, the set of states is formed by individual states of $\mathrm{HN}, \mathrm{HS} 1, \mathrm{HS} 2$ and combinations of these states for HN+HS1, HN+HS2 and HS1+HS2. The graph of DHS states in a reduced form is presented in Fig. 3. The number of component of the graph presented in Fig. 3 corresponds to the number of a failed component (components 1-18 are network sections, components 19-30 correspond to the sources). Markov random process that meets the specified conditions is described by the system consisting of 283 stationary linear equations of form (14).

Table 1. Technical characteristics of components of considered DHS (corresponding to scheme on Fig. 1)

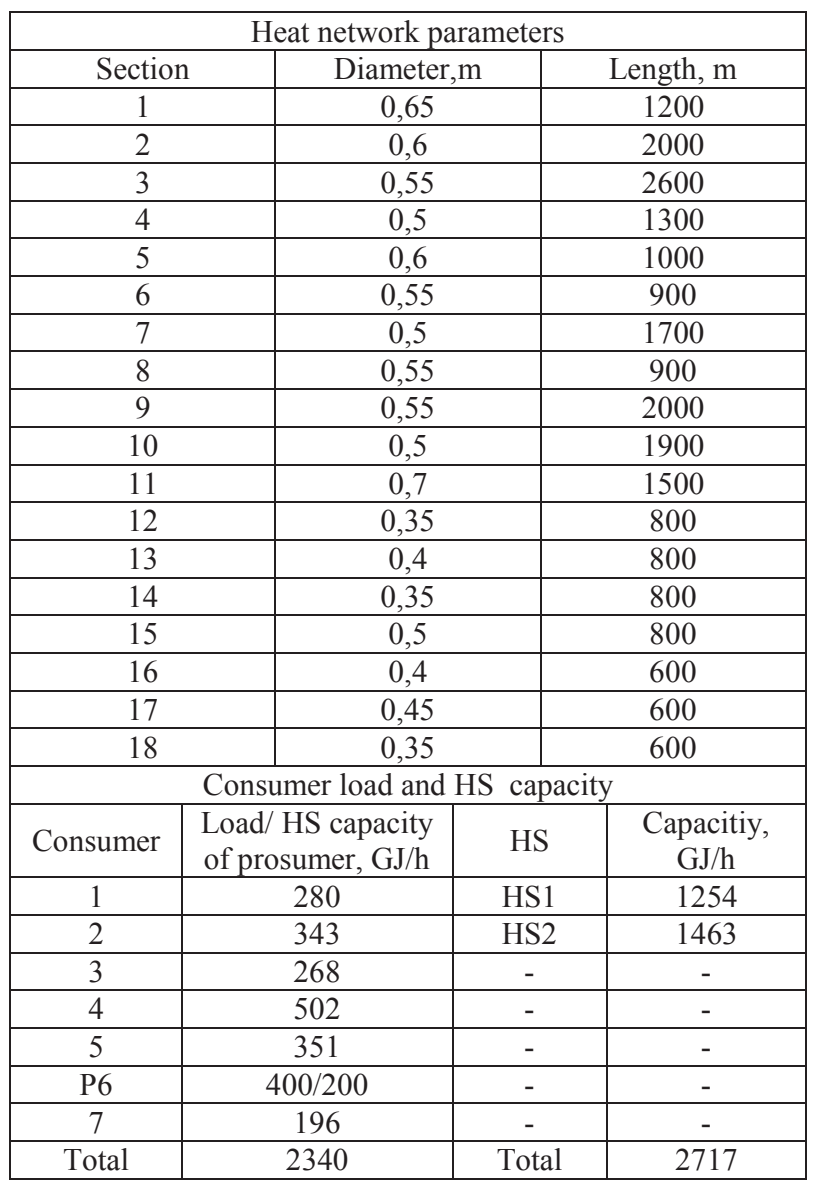

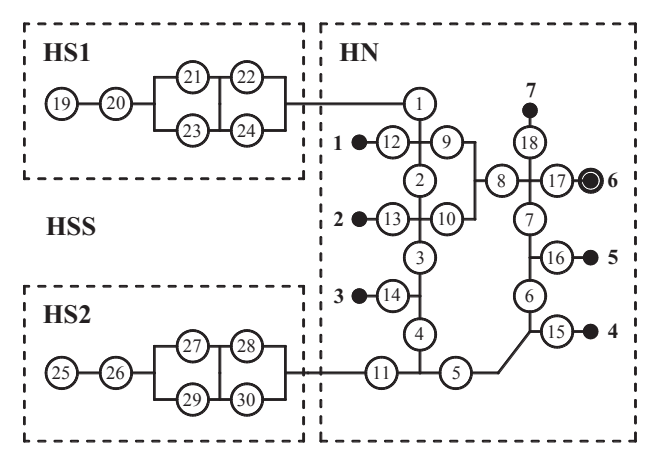

Fig. 2. Calculation scheme of HSS for reliability analysis (integrating component of schemes of HS and HN) 
The level of heat carrier delivery to consumers in different states of the DHS is determined by multivariant calculations of flow distribution in the $\mathrm{HN}$, considering the failures of HS equipment, with the model (9)-(11). The results of the calculation of probabilities of system states and post-emergency hydraulic conditions are not presented due to a large data array.

The reliability parameters of the DHS are optimized provided the following standard values of nodal reliability parameters [10] are met: $K_{\mathrm{oj}}=0,97$ and $R_{\mathrm{o} j}=$ 0,905 . The following ranges of possible values of optimized reliability parameters of the DHS components are assumed: $0,0002-0,00251 / \mathrm{h}$ - for failure rate; $0,007-0,091 / \mathrm{h}-$ for restoration rate.

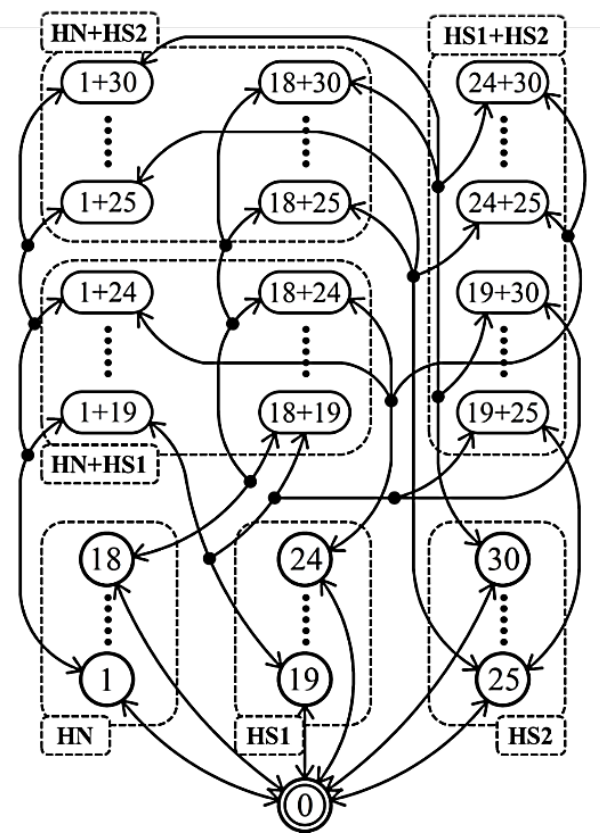

Fig. 3. Graph of HSS states and relation between them

Analytical functions of the costs required to restore the components of heat supply system (15) are assumed in an aggregate form, using the data on composition and specific costs of backup components of DHS, as well as the data on the establishment and maintenance of the ERS [10]. Analysis of these data made it possible using the approximation methods to obtain the required relationships between the costs and reliability parameters in the form of power objective functions.

Table 2 presents the calculated optimal average reliability parameters of DHS components and corresponding total costs of ensuring reliability of heat supply to consumers. There are two results for the costs: without prosumer P6 and with prosumer P6.

Fig. 4 illustrates a search for an optimal relationship between the average reliability parameters of DHS components that correspond to the minimum costs of ensuring reliability in the system when the required reliability indices (FOP and AF) are met. The presented diagram demonstrates three graphs: line 1 represents a relationship between the average reliability parameters of components, and graphs 2 and 3 show a change in the costs of ensuring the required reliability level in the DHS depending on the relationship between the average reliability parameters of components with and without HS of prosumer (P6), respectively.

Table 2. Result of optimization reliability parameters of HSS components (corresponding to diagram on Fig. 4)

\begin{tabular}{|c|c|c|c|}
\hline \multicolumn{2}{|c|}{$\begin{array}{c}\text { Average reliability } \\
\text { parameters of DHS } \\
\text { components, } 1 / h\end{array}$} & \multicolumn{2}{c|}{$\begin{array}{c}\text { Total costs of ensuring } \\
\text { required reliability of HSS, } \\
\text { million rub }\end{array}$} \\
\hline failure rate & $\begin{array}{c}\text { restoration } \\
\text { rate }\end{array}$ & $\begin{array}{c}\text { without } \\
\text { prosumer P6 }\end{array}$ & $\begin{array}{c}\text { with } \\
\text { prosumer P6 }\end{array}$ \\
\hline 0.0007 & 0.01 & 30.4 & 30.34 \\
\hline 0.0008 & 0.02 & 28.17 & 27.64 \\
\hline 0.0009 & 0.03 & 26.82 & 25.83 \\
\hline 0.0011 & 0.04 & 25.92 & 24.74 \\
\hline 0.0013 & 0.05 & 25.35 & 24.19 \\
\hline 0.0015 & 0.06 & 25.25 & 24.1 \\
\hline 0.0019 & 0.07 & 25.31 & 24.38 \\
\hline 0.0024 & 0.08 & 25.7 & 24.98 \\
\hline 0.0031 & 0.09 & 26.38 & 25.89 \\
\hline 0.0044 & 0.1 & 27.28 & 27.02 \\
\hline
\end{tabular}

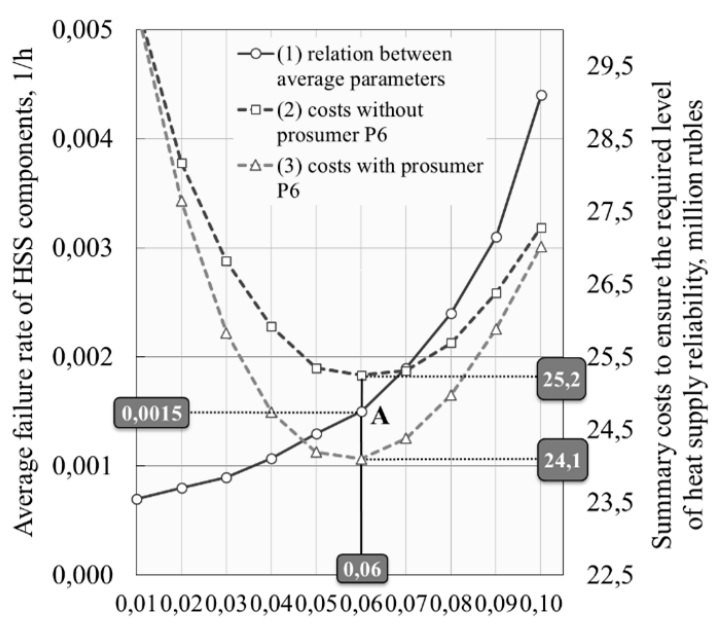

Average restoration rate of HSS components, $1 / \mathrm{h}$

Fig. 4. Search for an optimal relationship between average reliability parameters of HSS components

The obtained solution (point A) corresponds to the costs necessary to ensure reliability in the amount of 25,25 million rub without participation of HS of prosumer P6 and 24,1 million rub with the prosumer participation; i.e. an economic effect from the involvement of one prosumer in the considered system makes up 1.15 million rub, or $4.5 \%$. Thereby, the values of the average reliability parameters are: failure rate is $0.00151 / \mathrm{h}$, and restoration rate equals $0,061 / \mathrm{h}$.

\section{Conclusion}

The relevance of the research into heat prosumer is explained by objective heat supply problems related to low cost-effectiveness of operating systems, insufficient quality and reliability of heat supply to consumers, 
particularly due to technological development and growing loads.

The research formulates the statement of DHS reliability problem considering the prosumer functions as a way to provide additional capacity and time reserve owing to the prosumer HS. To solve this problem, we applied nodal reliability indices, Markov random process, models of the theory of hydraulic circuits and some basic laws of cogeneration and thermal-physical processes in heat supply.

The main advantage of the proposed technique lies in the integration of measures aimed at reducing the failures and enhancing restoration of the components in a joint procedure of search for reliability parameters, which makes it possible to most rationally distribute the total potential enabling the increase in reliability of DHS components.

The practical applicability of the developed methods for optimization of reliability parameters of DHS components is confirmed by calculations carried out on a test scheme. The calculation for the case with prosumer participation shows some economic effect and fulfillment of system reliability requirements.

The result obtained in the test calculations is not final to provide the rationale for the involvement of prosumer in ensuring the reliability of existing DHS. Further research aimed at a comprehensive analysis of the aspects of prosumer operation in the DHS (operation costs of prosumer sources, analysis of their operation, heat production cost cutting in the system, and other aspects) make it possible to formulate all the necessary criteria to substantiate the involvement of prosumers in DHS.

The research was performed at Melentiev Energy Systems Institute SB RAS under the support of Russian Science Foundation (Grant №17-19-01209).

\section{References}

1. Ni Zhang, Yu Yan, Wencong Su. Applied Energy, vol. 154, p. 471-479, (2015).

2. Luka Perkovic, Hrvoje Mikulcic, Neven Duic. Journal of Cleaner Production, xxx, p. 1-12, (2016).

3. Hongming Yang, Tonglin Xiong, Jing Qiu et al. Applied Energy, vol. 167, p. 353-365, (2016).

4. Rehman Zafar, Anzar Mahmood, Sohail Razzaq et al. Prosumer based energy management and sharing in smart grid // Renewable and Sustainable Energy Reviews, in press, available online 11 July 2017.

5. Stig Ødegaard Ottesen, Asgeir Tomasgard, Stein-Erik Fleten. Energy, vol. 94, p. 828-843, (2016).

6. Dimitrios J. Vergados, Ioannis Mamounakis, Prodromos Makris, Emmanouel Varvarigos. Sustainable Energy, Grids and Networks, vol. 7, p. 90-103, (2016).

7. Lakshmi Prakash, Sugatha Kumari P.R, Sharanya Chandran et al. Procedia Technology, vol. 21, p. 338344, (2015).

8. Lisa Brange, Jessica Englund, Patrick Lauenburg. Applied Energy, vol. 164, p. 492-500, (2016).
9. Lisa Brand, Alexandra Calvén, Jessica Englund et al. Applied Energy, vol. 129, p. 39-48, (2014).

10. Reliability of heat supply systems (Novosibirsk: Nauka, 2000. -360 p).

11. E.V. Sennova, V.G. Sidler. Mathematical modeling and optimization of developing heat supply systems (Novosibirsk, 1985. - $222 \mathrm{p}$ ).

12. V.Y. Sokolov District heating and heat networks (M.: MEI Publishing House, 1999. - 472 p).

13. A.P. Merenkov V.Y. Khasilev Theory of hydraulic circuits. (M.: Nauka, 1985. -278 p).

14. Reference book on general models for analysis and synthesis of energy system reliability/ Ed. by Y.N.Rudenko. - M.: Energoatomizdat, 1994. -474 p.

15. Chervonny A.A., Lukiyashchenko V.I., Kotin L.V. Reliability of complex systems. 2nd revised and corrected edition. (Mashinostroenie, 1976. - 288 p).

16. A.M. Plovko, S.V. Gurov Foundations of the reliability theory. (St. P.: BHV-Peterburg, 2006. - 702 p).

17. V.A. Kashtanov, A.I. Medvedev Theory of reliability of complex systems. (M.:Fizmatlit, 2010. - 608 p).

18. V.A. Stennikov, I.V. Postnikov Izv. RAN, Energetika. No.2, M.: Nauka, p.107-121, (2011).

19. V.A. Stennikov, I.V. Postnikov Power Technology and Engineering. Vol. 47, No. 6, March, p. 446-453, (2014).

20. V.A. Stennikov, I.V. Postnikov Sustaining power resources through energy optimization and engineering, Vasant P., Voropai N.I., editors. - Hershey PA: Engineering Science Reference (an imprint of IGI Global), p. 102-126, (2016).

21. Ivan V. Postnikov, Valery A. Stennikov, Ekaterina E. Mednikova, Andrey V. Penkovskii. Energy Procedia 105, P. 3083-3088. (2017).

22. Ekaterina E. Mednikova, Valery A. Stennikov, Ivan V. Postnikov. Energy Procedia 105, P. 3152-3157, (2017). 